\title{
Vibration analysis of rectangular plates with free corners using spline-based differential quadrature
}

\author{
Hongzhi Zhong* and Qiang Guo \\ Department of Civil Engineering, Tsinghua University, Beijing 100084, P. R. China
}

Received 7 May 2003

Accepted 24 September 2003

\begin{abstract}
A spline-based differential quadrature method (SDQM) is elaborated and applied to the vibration analysis of rectangular plates with free edges. The sextic B-spline functions are used to construct the pertaining cardinal spline interpolants. Thus, explicit expressions of weighting coefficients for approximation of derivatives are obtained. Free vibrations of rectangular plates with free edges, which pose considerable challenge to the conventional differential quadrature, are dealt with and the results are in excellent agreement with those in the literature, highlighting the effectiveness and potential of the spline-based differential quadrature.
\end{abstract}

\section{Introduction}

The differential quadrature method (DQM) has been applied to the analysis of various structural components such as beams, trusses, plates and shells [1]. Due to its simplicity and high efficiency, it has gained significant development especially over the past two decades. One of the key issues of the method is how to determine the weighting coefficients. So far, various basis functions have been used to determine the weighting coefficients among which the Lagrange interpolants are widely used for its simplicity and explicitness [2,3]. A wealth of information on the evaluation of the weighting coefficients and the discrete points for the DQM has been summarized by Bert and Malik [1], Shu and Chen [4,5] and Fung [6]. It has been widely recognized that the use of the Gauss-Lobatto-Chebyshev points [5] in the differential quadrature approximation can accelerate the convergence rate in majority cases, to which the success of the differential quadrature method is believed to be ascribed.

\footnotetext{
*Corresponding author. Fax: +86 10 62771132; E-mail: hzz@ mail.tsinghua.edu.cn.
}

The solution of free vibrations of rectangular plates was initiated by Bert and his associates [7,8]. As indicated in the work of Malik and Bert [9], the conventional differential quadrature solution of some rectangular plates with two and more than two free edges did not exhibit convergence trend and erratic results were obtained. Shu, et al. [10] attempted to improve the accuracy of the differential quadrature solution through the stretching of grid towards the boundary. But the stretching parameter $\alpha$ varies irregularly in different cases and therefore renders their approach inconvenient when dealing with practical problems. Karami and Malekzadeh have also dealt with this problem using the DQM [11].

The use of spline functions to determine the weighting coefficients in the differential quadrature method was actually initiated by Kashef and Bellman [12]. In their work, the weighting coefficients were determined using cubic B-spline functions, but they did not elaborate on how the weighting coefficients were evaluated. Furthermore, it is clear that the differential quadrature based on cubic B-spline functions is not applicable to the solution of high order differential equations. In this paper, a differential quadrature method based on sextic 
B-spline functions is enunciated and used to deal with free vibrations of rectangular plates. Since the free vibrations of rectangular plates with free corners poses challenge to the differential quadrature method, this paper will focus on the cases where the conventional differential quadrature method runs into difficulty [9]. It is shown that the solution of free vibrations of rectangular plates with various combinations of free edges using the present spline-based differential quadrature method exhibits obvious convergence trend. The comparison of computed results with the analytical solutions [13] indicates that the differential quadrature method based on the sextic B-spline functions is reliable and effective.

\section{Spline-based differential quadrature}

\subsection{Cardinal sextic spline interpolation}

In addition to tackling the challenging problems, a major motivation of the development of differential quadrature using the sextic B-spline is that the convergence rate of the differential quadrature method based on the quintic B-spline has been found to be less satisfactory in the case of vibration analysis of BernoulliEuler beams which is also characterized by fourth-order system. First of all, a set of uniformly spaced knots is selected in a normalized interval $[0,1]$, i.e.

$$
\begin{aligned}
x_{0} & =0, x_{N}=1, x_{j+1}-x_{j}=h, \\
j & =0,1,2, \ldots, N-1
\end{aligned}
$$

where $N+1$ is the number of knots in the interval, $h$ the length of every segment. The normalized sextic B-spline function is given by [14]

$$
\begin{gathered}
\varphi_{6}(x)=\frac{1}{6 ! h^{6}} \sum_{j=0}^{7}(-1)^{j}\left(\begin{array}{l}
7 \\
j
\end{array}\right)\left[x-\left(j-\frac{7}{2}\right) h\right]^{6} \\
\Delta\left[x-\left(j-\frac{7}{2}\right) h\right]
\end{gathered}
$$

where $\left(\begin{array}{l}7 \\ j\end{array}\right)$ are the binomial coefficients, and $\Delta(x)$ is the unit step function. Apparently, it is a piecewise polynomial that covers eight consecutive segments only. To construct a global interpolant over the interval, usually extra knots outside the interval $\left[x_{0}, x_{N}\right]$ are needed to meet the end condition requirements. A typical spline interpolation over the given interval using the sextic B-spline can be expressed in the form

$$
\begin{aligned}
& S_{6}(x)=\sum_{j=-3}^{N+3} \Phi_{j}(x) y_{j}, \\
& \Phi_{j}(x)=\Phi_{0}(x-j h)
\end{aligned}
$$

In order to meet the required interpolation condition

$$
S_{6}\left(x_{j}\right)=y_{i}
$$

the interpolants $\Phi_{j}(x)$ should satisfy the cardinal condition at every knot, i.e.

$$
\begin{aligned}
& \Phi_{j}\left(x_{i}\right)=\delta_{i j}=\left\{\begin{array}{l}
1 i=j ; \\
0, \text { otherwise. }
\end{array}\right. \\
& i, j=-3,-2,-1,0,1, \ldots, N-1 \text {, } \\
& N, N+1, N+2, N+3
\end{aligned}
$$

where $\Phi_{j}(x)$ are usually given in terms of a combination of translated and scaled spline function $\varphi_{6}$. To acquire the cardinal spline interpolant $\Phi_{j}(x)$, the following four auxiliary spline interpolants [15] are constructed first

$$
\begin{aligned}
& \psi_{6}(x)=\sum_{j=-3}^{N+3} y_{j} \varphi_{6}\left(x-x_{j}\right) \\
& \psi_{6}(x)^{<\frac{1}{6}>}=\sum_{j=-3}^{N+3} y_{j} \varphi_{6}^{<\frac{1}{6}>}\left(x-x_{j}\right) \\
& \psi_{6}(x)^{<\frac{1}{3}>}=\sum_{j=-3}^{N+3} y_{j} \varphi_{6}^{<\frac{1}{3}>}\left(x-x_{j}\right) \\
& \psi_{6}(x)^{<\frac{1}{2}>}=\sum_{j=-3}^{N+3} y_{j} \varphi_{6}^{<\frac{1}{2}>}\left(x-x_{j}\right)
\end{aligned}
$$

where

$$
\begin{aligned}
& \varphi_{6}^{<\frac{1}{6}>}(x) \equiv \varphi_{6}\left(x+\frac{h}{6}\right)+\varphi_{6}\left(x-\frac{h}{6}\right) \\
& \varphi_{6}^{<\frac{1}{3}>}(x) \equiv \varphi_{6}\left(x+\frac{h}{3}\right)+\varphi_{6}\left(x-\frac{h}{3}\right) \\
& \varphi_{6}^{<\frac{1}{2}>}(x) \equiv \varphi_{6}\left(x+\frac{h}{2}\right)+\varphi_{6}\left(x-\frac{h}{2}\right)
\end{aligned}
$$

With the local non-zero property of the spline function $\varphi_{6}(x)$, all the terms but the one containing $y_{j}$ on the right sides of Eq. (6) are eliminated. Thus, the cardinal spline interpolant is obtained as 


$$
\begin{aligned}
S_{6}(x)= & \frac{76946}{15} \psi_{6}(x)-\frac{152469}{40} \psi_{6}^{<\frac{1}{6}>}(x) \\
& +1485 \psi_{6}^{<\frac{1}{3}>}(x)-\frac{28517}{120} \psi_{6}^{<\frac{1}{2}>}(x),(8)
\end{aligned}
$$$$
S_{6}(x) \in C^{5}
$$

Hence,

$$
\begin{aligned}
\Phi_{j}(x)= & \frac{76946}{15} \varphi_{6}\left(x-x_{j}\right)-\frac{152469}{40} \varphi_{6}^{<\frac{1}{6}>} \\
& \left(x-x_{j}\right)+1485 \varphi_{6}^{<\frac{1}{3}>}\left(x-x_{j}\right) \\
& -\frac{28517}{120} \varphi_{6}^{<\frac{1}{2}>}\left(x-x_{j}\right)
\end{aligned}
$$

In Eq. (8), $C^{5}$ denotes that the derivatives of the function are continuous up to the quintic order. It can be shown readily that Eq. (4) is satisfied at every knot.

Since the extra knots outside the interval are often cumbersome to handle, non-integral knots within the interval are introduced instead in this paper. Namely, $x_{1 / 6}=h / 6, x_{1 / 3}=h / 3, x_{1 / 2}=h / 2$ and $x_{N-1 / 2}=$ $(N-1 / 2) h, x_{N-1 / 3}=(N-1 / 3) h, x_{N-1 / 6}=(N-$ $1 / 6) h$, are added in the vicinity of the two ends of the interval. Moreover, the function values at the nonintegral knots can be given by

$$
\begin{aligned}
y_{1 / 6}= & \sum_{i=-3}^{N+3} \Phi_{j}\left(\frac{h}{6}\right) y_{j} \\
= & \alpha_{11} y_{-3}+\alpha_{12} y_{-2}+\alpha_{13} y_{-1} \\
& +\alpha_{14} y_{0}+\alpha_{15} y_{1}+\alpha_{16} y_{2} \\
& +\alpha_{17} y_{3}+\alpha_{18} y_{4} \\
y_{1 / 3}= & \sum_{i=-3}^{N+3} \Phi_{j}\left(\frac{h}{3}\right) y_{j} \\
= & \alpha_{21} y_{-3}+\alpha_{22} y_{-2}+\alpha_{23} y_{-1} \\
& +\alpha_{24} y_{0}+\alpha_{25} y_{1}+\alpha_{26} y_{2} \\
& +\alpha_{27} y_{3}+\alpha_{28} y_{4} \\
& \\
y_{1 / 2}= & \sum_{i=-3}^{N+3} \Phi_{j}\left(\frac{h}{2}\right) y_{j} \\
= & \alpha_{31} y_{-3}+\alpha_{32} y_{-2}+\alpha_{33} y_{-1} \\
& +\alpha_{34} y_{0}+\alpha_{35} y_{1}+\alpha_{36} y_{2} \\
& +\alpha_{37} y_{3}+\alpha_{38} y_{4}
\end{aligned}
$$

where $\alpha_{i j}(i=1,2,3 ; j=1, \ldots, 8)$ are constants which are given in the Appendix of the paper. Then, the function values at the extra knots outside the left end of the interval can be obtained by solving Eqs (10a), (10b) and (10c)

$$
\begin{aligned}
y_{-1}= & \beta_{11} y_{0}+\beta_{12} y_{1 / 6}+\beta_{13} y_{1 / 3}+\beta_{14} y_{1 / 2} \\
& +\beta_{15} y_{1}+\beta_{16} y_{2}+\beta_{17} y_{3}+\beta_{18} y_{4}
\end{aligned}
$$

$$
\begin{aligned}
y_{-2}= & \beta_{21} y_{0}+\beta_{22} y_{1 / 6}+\beta_{23} y_{1 / 3}+\beta_{24} y_{1 / 2} \\
& +\beta_{25} y_{1}+\beta_{26} y_{2}+\beta_{27} y_{3}+\beta_{28} y_{4}
\end{aligned}
$$

$$
\begin{aligned}
y_{-3}= & \beta_{31} y_{0}+\beta_{32} y_{1 / 6}+\beta_{33} y_{1 / 3}+\beta_{34} y_{1 / 2} \\
& +\beta_{35} y_{1}+\beta_{36} y_{2}+\beta_{37} y_{3}+\beta_{38} y_{4}
\end{aligned}
$$

where $\beta_{i j}(i=1,2,3 ; j=1, \ldots, 8)$ are constants also given in the Appendix of the paper. Similarly, the function values at the extra knots outside the right end of the interval are expressed as

$$
\begin{aligned}
y_{N+1}= & \beta_{11} y_{N}+\beta_{12} y_{N-1 / 6}+\beta_{13} y_{N-1 / 3} \\
& +\beta_{14} y_{N-1 / 2}+\beta_{15} y_{N-1}+\beta_{16} y_{N-2} \\
& +\beta_{17} y_{N-3}+\beta_{18} y_{N-4}
\end{aligned}
$$

$$
\begin{aligned}
y_{N+2}= & \beta_{21} y_{N}+\beta_{22} y_{N-1 / 6}+\beta_{23} y_{N-1 / 3} \\
& +\beta_{24} y_{N-1 / 2}+\beta_{25} y_{N-1}+\beta_{26} y_{N-2} \\
& +\beta_{27} y_{N-3}+\beta_{28} y_{N-4}
\end{aligned}
$$

$$
\begin{aligned}
y_{N+3}= & \beta_{31} y_{N}+\beta_{32} y_{N-1 / 6}+\beta_{33} y_{N-1 / 3} \\
& +\beta_{34} y_{N-1 / 2}+\beta_{35} y_{N-1}+\beta_{36} y_{N-2} \\
& +\beta_{37} y_{N-3}+\beta_{38} y_{N-4}
\end{aligned}
$$

Now, the cardinal sextic spline interpolant can be re-arranged into the following form that is free of extra outside knots

$$
\begin{aligned}
s_{6}(x)= & \sum_{j=0}^{N} \Omega_{j}(x) y_{j}, \Omega_{j}\left(x_{i}\right) \\
& =\delta_{i j}=\left\{\begin{array}{l}
1, i=j ; \\
0, \text { otherwise. }
\end{array}\right. \\
i, j= & 0,1 / 6,1 / 3,1 / 2,1,2, \ldots, N-2, N-1, \\
& \quad N-1 / 2, N-1 / 3, N-1 / 6, N
\end{aligned}
$$

where 


$$
\begin{aligned}
\Omega_{0}(x)= & \beta_{31} \Phi_{-3}(x)+\beta_{21} \Phi_{-2}(x) \\
& +\beta_{11} \Phi_{-1}(x)+\Phi_{0}(x) \\
\Omega_{1 / 6}(x)= & \beta_{32} \Phi_{-3}(x)+\beta_{22} \Phi_{-2}(x) \\
& +\beta_{12} \Phi_{-1}(x) \\
\Omega_{1 / 3}(x)= & \beta_{33} \Phi_{-3}(x)+\beta_{23} \Phi_{-2}(x) \\
& +\beta_{13} \Phi_{-1}(x) \\
\Omega_{1 / 2}(x)= & \beta_{34} \Phi_{-3}(x)+\beta_{24} \Phi_{-2}(x) \\
& +\beta_{14} \Phi_{-1}(x) \\
\Omega_{1}(x)= & \beta_{35} \Phi_{-3}(x)+\beta_{25} \Phi_{-2}(x) \\
& +\beta_{15} \Phi_{-1}(x)+\Phi_{1}(x) \\
\Omega_{2}(x)= & \beta_{36} \Phi_{-3}(x)+\beta_{26} \Phi_{-2}(x) \\
& +\beta_{16} \Phi_{-1}(x)+\Phi_{2}(x) \\
\Omega_{3}(x)= & \beta_{37} \Phi_{-3}(x)+\beta_{27} \Phi_{-2}(x) \\
& +\beta_{17} \Phi_{-1}(x)+\Phi_{3}(x) \\
\Omega_{4}(x)= & \beta_{38} \Phi_{-3}(x)+\beta_{28} \Phi_{-2}(x) \\
& +\beta_{18} \Phi_{-1}+\Phi_{4}(x)
\end{aligned}
$$

and

$$
\Omega_{i}(x)=\Phi_{i}(x), \text { for } 5 \leqslant i \leqslant N-5 ;
$$

and

$$
\begin{aligned}
\Omega_{N-4}(x)= & \beta_{38} \Phi_{N+3}(x)+\beta_{28} \Phi_{N+2}(x) \\
& +\beta_{18} \Phi_{N+1}(x)+\Phi_{N-4}(x) \\
\Omega_{N-3}(x)= & \beta_{37} \Phi_{N+3}(x)+\beta_{27} \Phi_{N+2}(x) \\
& +\beta_{17} \Phi_{N+1}(x)+\Phi_{N-3}(x) \\
\Omega_{N-2}(x)= & \beta_{36} \Phi_{-3}(x)+\beta_{26} \Phi_{N+2}(x) \\
& +\beta_{16} \Phi_{N+1}(x)+\Phi_{N-2}(x) \\
\Omega_{N-1}(x)= & \beta_{35} \Phi_{N+3}(x)+\beta_{25} \Phi_{N+2}(x) \\
& +\beta_{15} \Phi_{N+1}(x)+\Phi_{N-1}(x) \\
\Omega_{N-1 / 2}(x)= & \beta_{34} \Phi_{N+3}(x)+\beta_{24} \Phi_{N+2}(x) \\
& +\beta_{14} \Phi_{N+1}(x) \\
\Omega_{N-1 / 3}(x)= & \beta_{33} \Phi_{N+3}(x)+\beta_{23} \Phi_{N+2}(x) \\
& +\beta_{13} \Phi_{N+1}(x) \\
\Omega_{N-1 / 6}(x)= & \beta_{32} \Phi_{N+3}(x)+\beta_{22} \Phi_{N+2}(x) \\
& +\beta_{12} \Phi_{N+1}(x) \\
\Omega_{N}(x)= & \beta_{31} \Phi_{N+3}(x)+\beta_{21} \Phi_{N+2}(x) \\
& +\beta_{11} \Phi_{N+1}(x)+\Phi_{N}(x)
\end{aligned}
$$

\subsection{Weighting coefficients}

The essence of the differential quadrature is that the derivative of a function with respect to a space variable at a given point is approximated by a weighted linear summation of the function values at all discrete points in the domain. Therefore, the approximation of a derivative at a knot in spline-based differential quadrature is given by

$$
\begin{aligned}
D_{n}\{f(x)\}_{i}= & \sum_{j=0}^{N} C_{i j}^{(n)} f\left(x_{j}\right) \\
i, j= & 0,1 / 6,1 / 3,1 / 2,1,2, \ldots, \\
& N-2, N-1, N-1 / 2, \\
& N-1 / 3, N-1 / 6, N
\end{aligned}
$$

where $D_{n}$ is a differential operator of order $n$, the subscript indicates the value of $D_{n}\{f(x)\}$ at $\operatorname{knot} x_{i}, C_{i j}^{(n)}$, are the weighting coefficients related to the function values . If it is required that Eq. (15) be exactly satisfied when function $f(x)$ takes the cardinal spline basis functions $\Omega_{j}(x)$, all weighting coefficients are given in explicit forms and computed analytically.

$$
\begin{aligned}
C_{i j}^{(n)} & =\Omega_{j}^{(n)}\left(x_{i}\right), n=1, \ldots, 6 \\
i, j & =0,1 / 6,1 / 3,1 / 2,1,2, \ldots, N-2,
\end{aligned}
$$

$$
N-1, N-1 / 2, N-1 / 3, N-1 / 6, N
$$

The localized non-zero nature of splines results in banded weighting coefficient matrices for derivatives. Meanwhile, the following relationships among weighting coefficients exist

$$
\begin{aligned}
& C_{i j}^{(1)}=-C_{[(N-i)(N-j)}^{(1)}, C_{i j}^{(3)}=-C_{(N-i)(N-j)}^{(3)}, \\
& C_{i j}^{(5)}=-C_{(N-1)(N-j)}^{(5)}, \\
& C_{i j}^{(2)}=C_{(N-i)(N-j)}^{(2)}, C_{i j}^{(4)}=C_{(N-i)(N-j)}^{(4)}, \\
& C_{i j}^{(6)}=C_{(N-i)(N-j)}^{(6)} .
\end{aligned}
$$

\section{Vibrations of thin rectangular plates}

The free vibration analysis of thin rectangular plates of isotropic materials involves essentially the solution of the well-known governing differential equation [9]:

$$
\begin{aligned}
& w_{, X X X X}+2 \lambda^{2} w_{, X X Y Y}+\lambda^{4} w_{, Y Y Y Y} \\
& -\Omega^{2} w=0,
\end{aligned}
$$


where $w=w(X, Y)$ is the deflection mode corresponding to the dimensionless frequency $\Omega ; X=$ $x / a, Y=y / b$ are dimensionless coordinates; $a$ and $b$ are the lengths of the plate edges parallel to the $x$ and $y$ axes, respectively; and $\lambda=a / b$ is the aspect ratio. Further, $\Omega=\omega a^{2} \sqrt{\rho h / D}$, where $\omega$ is the dimensional circular frequency; $D=E h^{3} /\left[12\left(1-v^{2}\right)\right]$, the flexural rigidity; $E, v$, and $\rho$ are Young's modulus, Poisson's ratio, and the density of the plate material, respectively; and $h$ is the plate thickness. The subscripts in (18) stand for the partial differentiation, such as $w, X X X X=\partial^{4} w / \partial X^{4}$, etc.

In general, three typical boundary conditions are commonly used in engineering practice: simply supported edge, fully clamped edge and free edge, which are denoted as SS, C and F for simplicity of presentation, respectively. For a simply supported or a clamped boundary, the transverse deflection of the plate is zero:

$$
w=0 \text {. }
$$

At a simply supported boundary, the condition of zero normal moment can be reduced to

$$
w, X X=0
$$

at a $Y$-parallel edge, and

$$
w_{, Y Y}=0
$$

at an $X$-parallel edge.

At a clamped boundary, the condition of zero rotation is given by

$$
w_{, X}=0
$$

at a $Y$-parallel edge, and

$$
w_{, Y}=0
$$

at an $X$-parallel edge.

The condition of zero normal moment at a free boundary is given by

$$
w_{, X X}+v \lambda^{2} w_{, Y Y}=0
$$

at a $Y$-parallel edge, and

$$
\lambda^{2} w_{, Y Y}+v w, X X=0
$$

at an $X$-parallel edge.

The condition of zero effective shear force at a free boundary is given by

$$
w_{, X X X}+(2-v) \lambda^{2} w_{, X Y Y}=0
$$

at a $Y$-parallel edge, and

$$
\lambda^{2} w_{, Y Y Y}+(2-v) w_{, X X Y}=0
$$

at an $X$-parallel edge.
An additional condition that applies at a free corner that is formed by two adjacent free edges is given by

$$
w_{, X Y}=0 \text {. }
$$

An eigenvalue problem can be formulated if the governing differential Eq. (18) and the relevant boundary conditions are transformed into algebraic equations with aid of the quadrature analog (15). For convenience, the same number of knots is selected in the two directions, say, $N$. Adding in the non-integer knots in the two directions, there are $(N+7) \times(N+7)$ knots. The quadrature analogs of the differential Eq. (18) are implemented at $(N+3) \times(N+3)$ inner knots while boundary conditions including corner conditions are written at knots on the four edges and in the vicinity of the four edges. The details can be found in relevant publications $[1,16]$. Eventually, one arrives at algebraic equations of the following form

$$
\left[\begin{array}{l}
{\left[K_{b b}\right]\left[K_{b i}\right]} \\
{\left[K_{i b}\right]\left[K_{i i}\right]}
\end{array}\right]\left\{\begin{array}{l}
\left\{w_{b}\right\} \\
\left\{w_{i}\right\}
\end{array}\right\}=\left\{\begin{array}{c}
\{0\} \\
\Omega^{2}\left\{w_{i}\right\}
\end{array}\right\}
$$

where $\left\{w_{b}\right\}$ is the deflection vector at the four edges of the plate, $\left\{w_{i}\right\}$ the deflection vector at all inner knots. By eliminating the $\left\{w_{b}\right\}$ vector, the above equations become a standard eigenvalue problem of the size $(N+$ $3) \times(N+3)$, i.e.

$$
[K]\left\{w_{i}\right\}=\Omega^{2}\left\{w_{i}\right\}
$$

The eigen-values are extracted using a double QR algorithm [17].

\section{Numerical results and discussion}

To simplify presentation, all rectangular plates are designated by the boundary conditions in a clockwise (or anti-clockwise) sequence. For cases where the conventional differential quadrature solutions are satisfactory, the present spline-based differential quadrature solutions are satisfactory as well. Compared with the conventional DQM, the spline-based differential quadrature usually requires more points to obtain the same order of accuracy. This is attributed to the low order approximation of the spline functions. But the computational efficiency is not affected very much, for the resultant coefficient matrix is banded. In what follows, emphasis will be placed on the cases where the conventional differential quadrature method failed to yield results of dependable accuracy [9]. To validate the present spline-based differential quadrature method, the following five cases: F-F-F-F, S-F-F-F, C- 


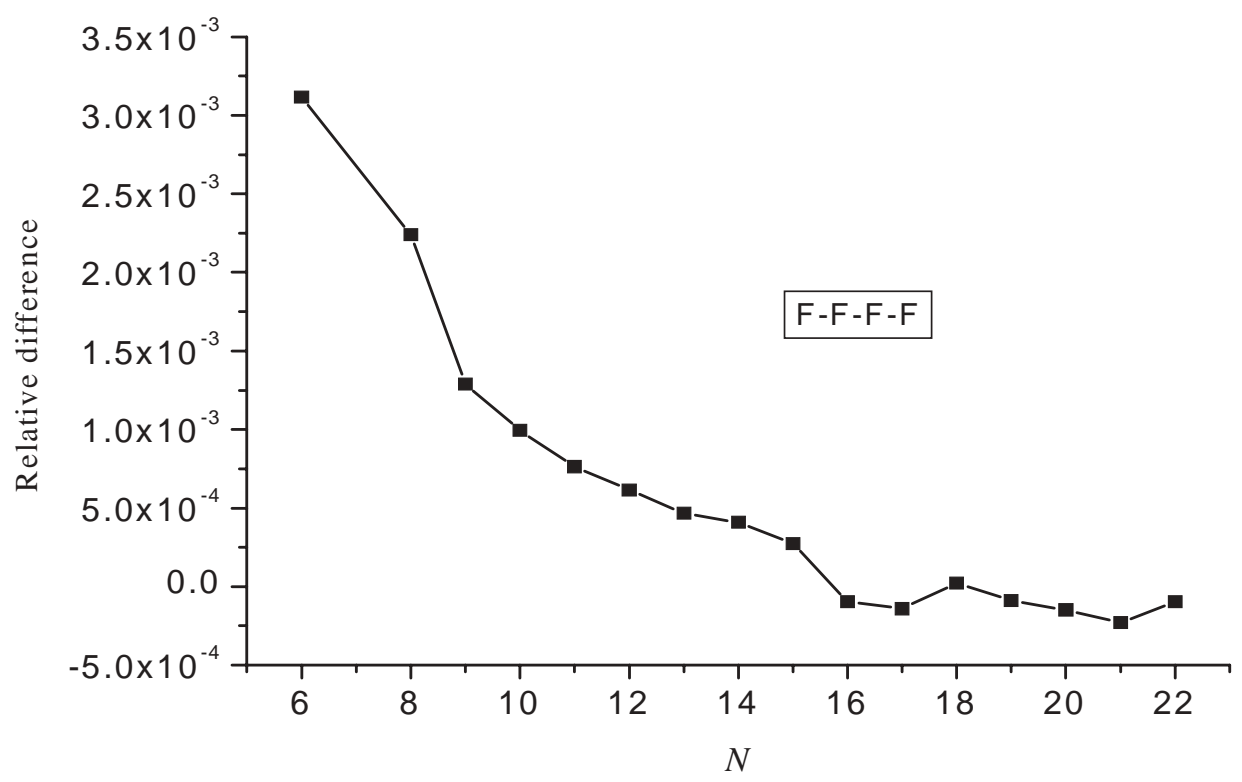

Fig. 1. Relative difference of fundamental frequency versus $\mathrm{N}$ for F-F-F-F plate.

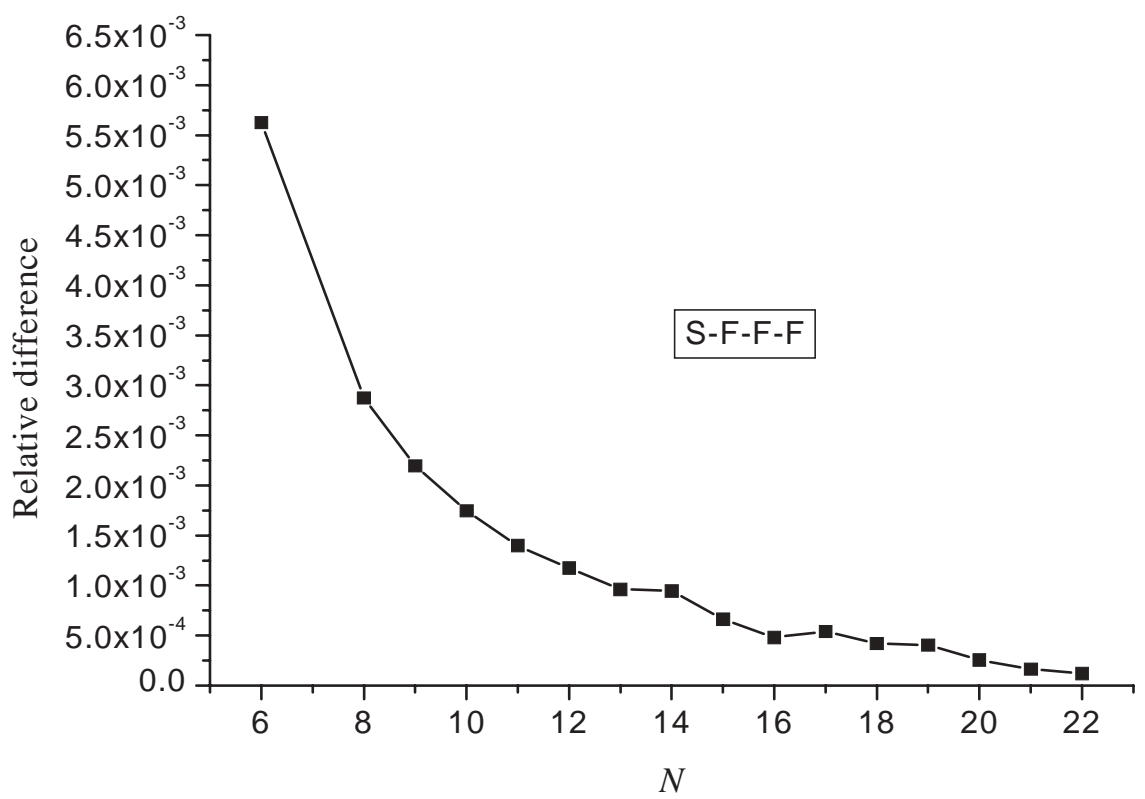

Fig. 2. Relative difference of fundamental frequency versus N for S-F-F-F plate.

F-F-F, C-S-F-F and C-C-F-F, which pose considerable difficulty to the conventional differential quadrature [9], are considered herein. Although the aspect ratio is a parameter that has to be considered in vibration analysis of plates, only square plates $(\lambda=1)$ are considered since they were recognized as the rather representative cases in vibration analysis of plates [13]. Furthermore, the differential quadrature solution is not sensitive to the effect of aspect ratio in so far as it is within a reasonable range. The Poisson's ratio is taken as 0.3 in all cases. To investigate the convergence of the present method, the first six linear natural frequency parameters for a square F-F-F-F plate are obtained and displayed in Table 1. For the fundamental frequency, excellent agreement with the analytical value is reached. For the next five frequency parameters, all the maximum 


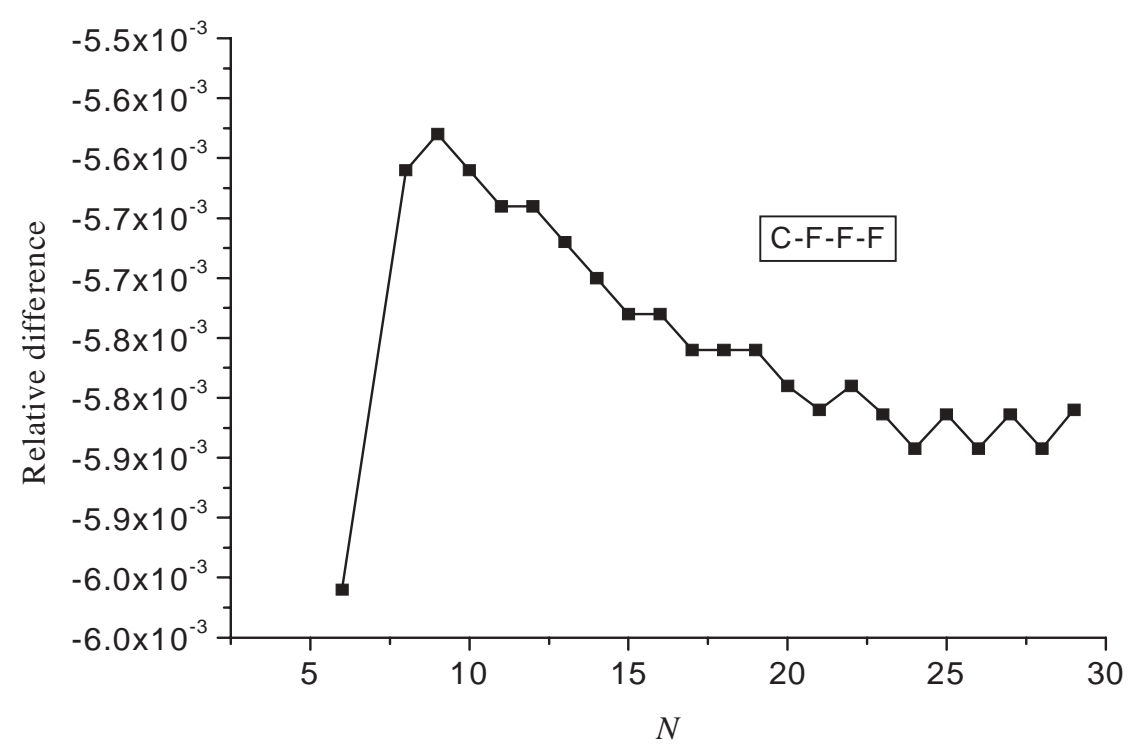

Fig. 3. Relative difference of fundamental frequency versus $\mathrm{N}$ for C-F-F-F plate.

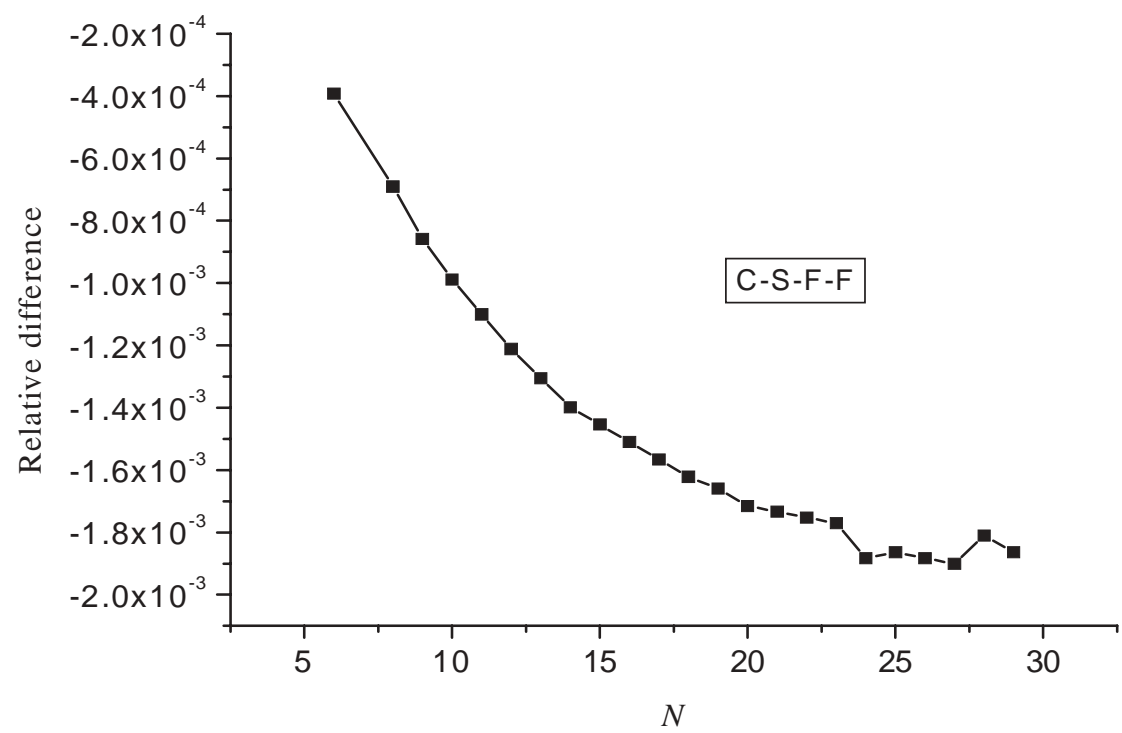

Fig. 4. Relative difference of fundamental frequency versus $\mathrm{N}$ for C-S-F-F plate.

relative errors are less than $1 \%$ when $\mathrm{N}$ is larger than 6 , highlighting the remarkable efficacy of the present method.

Figures 1-5 display the relative differences of the fundamental frequency parameters versus $\mathrm{N}$ for the plates with the five aforementioned boundary conditions, respectively. To probe into the approximation trend closely, the relative difference is defined as

$$
e=\frac{S D Q M-\text { reference[13] }}{\text { reference[13] }}
$$

In all cases, the solutions of the present method have obvious convergence trend despite the presence of slight oscillations. The amplitudes of the oscillations have been found very small in comparison with the solution values. Especially for F-F-F-F and S-FF-F plates, the absolute relative difference of the fundamental frequency parameter can be kept below 1E4 , contrasting to the erratic results of the conventional differential quadrature solutions [9]. It is worth mentioning that the analytical results given by Leissa [13] 
Table 1

The first six linear natural frequency parameters for F-F-F-F plate

\begin{tabular}{ccccccc}
\hline \multirow{2}{*}{$\mathrm{N}$} & \multicolumn{7}{c}{ Mode sequence } \\
\cline { 2 - 7 } & 1 & 2 & 3 & 4 & 5 & 6 \\
\hline 6 & 13.5220 & 19.5879 & 24.2784 & 34.9440 & 34.9440 & 61.0542 \\
& $(0.24 \%)$ & $(-1.02 \%)$ & $(-0.63 \%)$ & $(-0.23 \%)$ & $(-0.23 \%)$ & $(-0.77 \%)$ \\
\multirow{2}{*}{8} & 13.5102 & 19.5932 & 24.2817 & 34.8833 & 34.8833 & 61.0888 \\
& $(0.16 \%)$ & $(-0.99 \%)$ & $(-0.62 \%)$ & $(-0.40 \%)$ & $(-0.40 \%)$ & $(-0.71 \%)$ \\
10 & 13.4934 & 19.5946 & 24.2801 & 34.8565 & 34.8565 & 61.0968 \\
& $(0.03 \%)$ & $(-0.98 \%)$ & $(-0.62 \%)$ & $(-0.48 \%)$ & $(-0.48 \%)$ & $(-0.70 \%)$ \\
11 & 13.4903 & 19.5935 & 24.2797 & 34.8483 & 34.8483 & 61.0980 \\
& $(0.01 \%)$ & $(-0.99 \%)$ & $(-0.62 \%)$ & $(-0.50 \%)$ & $(-0.50 \%)$ & $(-0.70 \%)$ \\
13 & 13.4863 & 19.5962 & 24.2788 & 34.8375 & 34.8375 & 61.0987 \\
& $(-0.02 \%)$ & $(-0.97 \%)$ & $(-0.63 \%)$ & $(-0.53 \%)$ & $(-0.53 \%)$ & $(-0.69 \%)$ \\
\multirow{2}{*}{18} & 13.4803 & 19.5946 & 24.2763 & 34.8228 & 34.8228 & 61.0984 \\
& $(-0.06 \%)$ & $(-0.98 \%)$ & $(-0.64 \%)$ & $(-0.57 \%)$ & $(-0.57 \%)$ & $(-0.69 \%)$ \\
Leissa $[13]$ & 13.489 & 19.789 & 24.432 & 35.024 & 35.024 & 61.526 \\
\hline
\end{tabular}

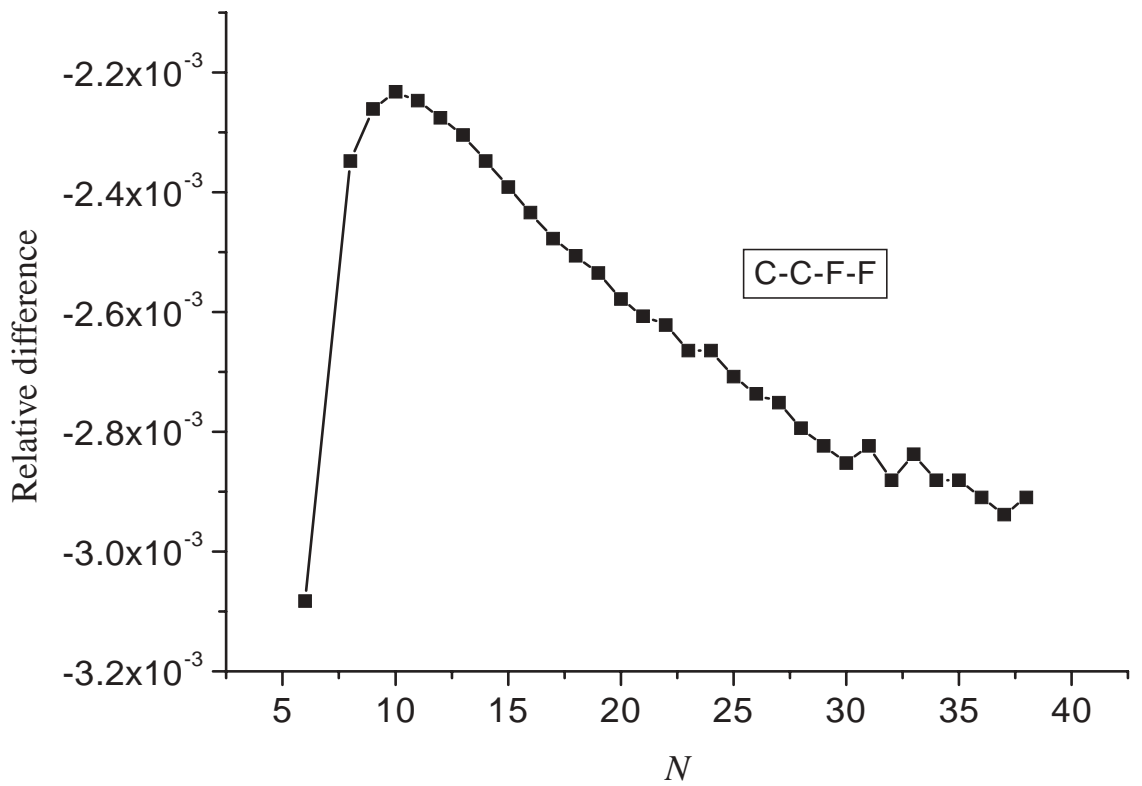

Fig. 5. Relative difference of fundamental frequency versus $\mathrm{N}$ for C-C-F-F plate.

are upper bounds of the exact values. Thus, the negative differences in Figures 3-5 may well imply that the present solutions are closer to the exact values of the fundamental frequencies than those in reference [13]. Among the five cases investigated, C-C-F-F is no doubt the most poorly behaved one. Interestingly, the same conclusion was drawn in analytical treatment of the case [9]. As shown in Fig. 5, larger $\mathrm{N}$ is needed to acquire convergent results for this case. An interpretation of the phenomenon could be the presence of sharply contrasted configurative features of the free corner and the clamped corner and the stringent diagonal symmetry.

\section{Conclusions}

Based on sextic cardinal spline functions, a splinebased differential quadrature method is presented. In the analysis of the free vibrations of plates with free edges, the computed results are found to be in very good agreement with those of the analytical results, indicating that the present method is effective. It is also shown that the spline-based differential quadrature method is very stable and has obvious superiority in some cases. It can deal with problems that pose great challenge to the conventional differential quadrature method. It is anticipated that the spline-based differential quadrature method can be extended to other problems in addition to the free vibrations of plates. 


\section{Acknowledgment}

The financial support of Fundamental Research Foundation of Tsinghua under Grant JC2001003 is gratefully acknowledged.

\section{Appendix}

The coefficients $\alpha_{i j}(i=1,2,3, j=1, \ldots 8)$ in Eq. (10)

$$
\begin{aligned}
& \alpha_{11}=-\frac{10181683}{4031078400}, \alpha_{12}=\frac{89335981}{4031078400}, \\
& \alpha_{13}=-\frac{138367381}{1343692800}, \alpha_{14}=\frac{775775581}{806215680} \\
& \alpha_{15}=\frac{116595899}{806215680}, \alpha_{16}=-\frac{35471219}{1343692800} \\
& \alpha_{17}=\frac{11611019}{4031078400}, \alpha_{18}=-\frac{28517}{4031078400} \\
& \alpha_{21}=-\frac{1944539}{503884800}, \alpha_{22}=\frac{16990973}{503884800}, \\
& \alpha_{23}=-\frac{25438973}{167961600}, \alpha_{24}=\frac{84574973}{100776960} \\
& \alpha_{25}=\frac{33697027}{100776960}, \alpha_{26}=-\frac{10042627}{167961600} \\
& \alpha_{27}=\frac{4129027}{503884800}, \alpha_{28}=-\frac{205861}{503884800} \\
& \alpha_{31}=-\frac{5}{2048}, \alpha_{32}=\frac{49}{2048}, \\
& \alpha_{37}=\frac{49}{2048}, \alpha_{38}=-\frac{5}{2045}, \alpha_{34}=\frac{1225}{2048} \\
& \alpha_{35}=\frac{1225}{2048}, \alpha_{36}=-\frac{245}{2048}, \\
& \alpha_{33}=-12
\end{aligned}
$$

The coefficients $\beta_{i j}(i=1,2,3, j=1, \ldots, 8)$ in Eqs (11), (12) and (14)

$$
\begin{aligned}
& \beta_{11}=\frac{17985004555}{131497139}, \\
& \beta_{12}=-\frac{481841986176}{1446468529}, \\
& \beta_{13}=\frac{304561981584}{1446468529}, \\
& \beta_{14}=\frac{1552804224}{131497139},
\end{aligned}
$$

$$
\begin{aligned}
& \beta_{15}=-\frac{3851757854}{131497139}, \\
& \beta_{16}=\frac{7533677746}{1446468529}, \\
& \beta_{17}=-\frac{137862319}{131497139}, \\
& \beta_{18}=\frac{162720709}{1446468529} \\
& \beta_{21}=\frac{161729840125}{131497139}, \\
& \beta_{22}=-\frac{4424269973760}{1446468529} \text {, } \\
& \beta_{23}=\frac{2443617447840}{1446468529} \\
& \beta_{24}=\frac{64964588288}{131497139}, \\
& \beta_{25}=-\frac{54970460440}{131497139} \text {, } \\
& \beta_{26}=\frac{114801658685}{1446468529}, \\
& \beta_{27}=-\frac{2216182024}{131497139}, \\
& \beta_{28}=\frac{2711690325}{1446468529} \\
& \beta_{31}=\frac{735904009085}{131497139} \\
& \beta_{32}=-\frac{19747588420224}{1446468529}, \\
& \beta_{33}=\frac{9023913891216}{1446468529}, \\
& \beta_{34}=\frac{506704330112}{131497139}, \\
& \beta_{35}=-\frac{317757578411}{131497139} \text {, } \\
& \beta_{36}=\frac{685029087638}{1446468529} \text {, } \\
& \beta_{37}=-\frac{13674658242}{131497139}, \\
& \beta_{38}=\frac{17154781915}{1446468529}
\end{aligned}
$$

\section{References}

[1] C.W. Bert and M. Malik, Differential quadrature method in computational mechanics: A Review, Applied Mechanics Reviews 49 (1996), 1-28. 
[2] J.R. Quan and C.T. Chang, New insights in solving distributed system equations by the quadrature method-I. Analysis, Computers and Chemical Engineering 13 (1989), 779-788.

[3] C. Shu and B.E. Richards, Application of generalized differential quadrature to solve two-dimensional incompressible Navier-Stokes equations, International Journal of Numerical Methods in Fluids 15 (1992), 791-798.

[4] C. Shu, Differential Quadrature and Its Application in Engineering, Spinger-Verlag, London, 2000.

[5] C. Shu and W. Chen, On optimal selection of interior points for applying discretized boundary conditions in DQ vibration analysis of beams and plates, Journal of Sound and Vibration 222 (1999), 239-257.

[6] T.C. Fung, Imposition of boundary conditions by modifying the weighting coefficient matrices in the differential quadrature method, International Journal for Numerical Methods in Engineering 56 (2003), 405-432.

[7] C.W. Bert, S.K. Jang and A.G. Striz, Two new approximate methods for analyzing free vibration of structural components, AIAA Journal 26 (1988), 612-618.

[8] S.K. Jang, C.W. Bert and A.G. Striz, Application of differential quadrature to static analysis of structural components, International Journal for Numerical Methods in Engineering 28 (1989), 561-577.

[9] M. Malik and C.W. Bert, Implementing multiple boundary conditions in the DQ solution of higher-order PDE's: application to free vibration of plates, International Journal for
Numerical Methods in Engineering 39 (1996), 1237-1258.

[10] C. Shu, W. Chen, H. Xue and H. Du, Numerical study of grid distribution effect on accuracy of DQ analysis of beams and plates by error estimation of derivative approximation, International Journal for Numerical Methods in Engineering 51 (2001), 159-179.

[11] G. Karamiand P. Malekzadeh, Application of a new differential quadrature methodology for free vibration analysis of plates, International Journal for Numerical Methods in Engineering 56 (2003), 847-868.

[12] B. Kashef and R. Bellman, Solution of the partial differential equation of Hodgkin-Huxley model using differential quadrature, Mathematical Biosciences 19 (1974), 1-8.

[13] A.W. Leissa, The free vibration of rectangular plates, Journal of Sound and Vibration 31 (1973), 257-293.

[14] L.L. Schumaker, Spline Functions: Basic Theory, Wiley, New York, 1981.

[15] Y.S. Li and D.X. Qi, Spline Function Method, Science Press, Beijing. 1979, in Chinese.

[16] M. Malik and C.W. Bert, Three-dimensional elasticity solutions for free vibrations of rectangular plates by the differential quadrature method, International Journal of Solids and Structures 35 (1998), 299-318.

[17] W.H. Press, B.P. Flannery, S.A. Teukolsky and W.T. Vetterling, Numerical Recipes-The Art of Scientific Computing, Cambridge University Press, Cambridge, 1986. 

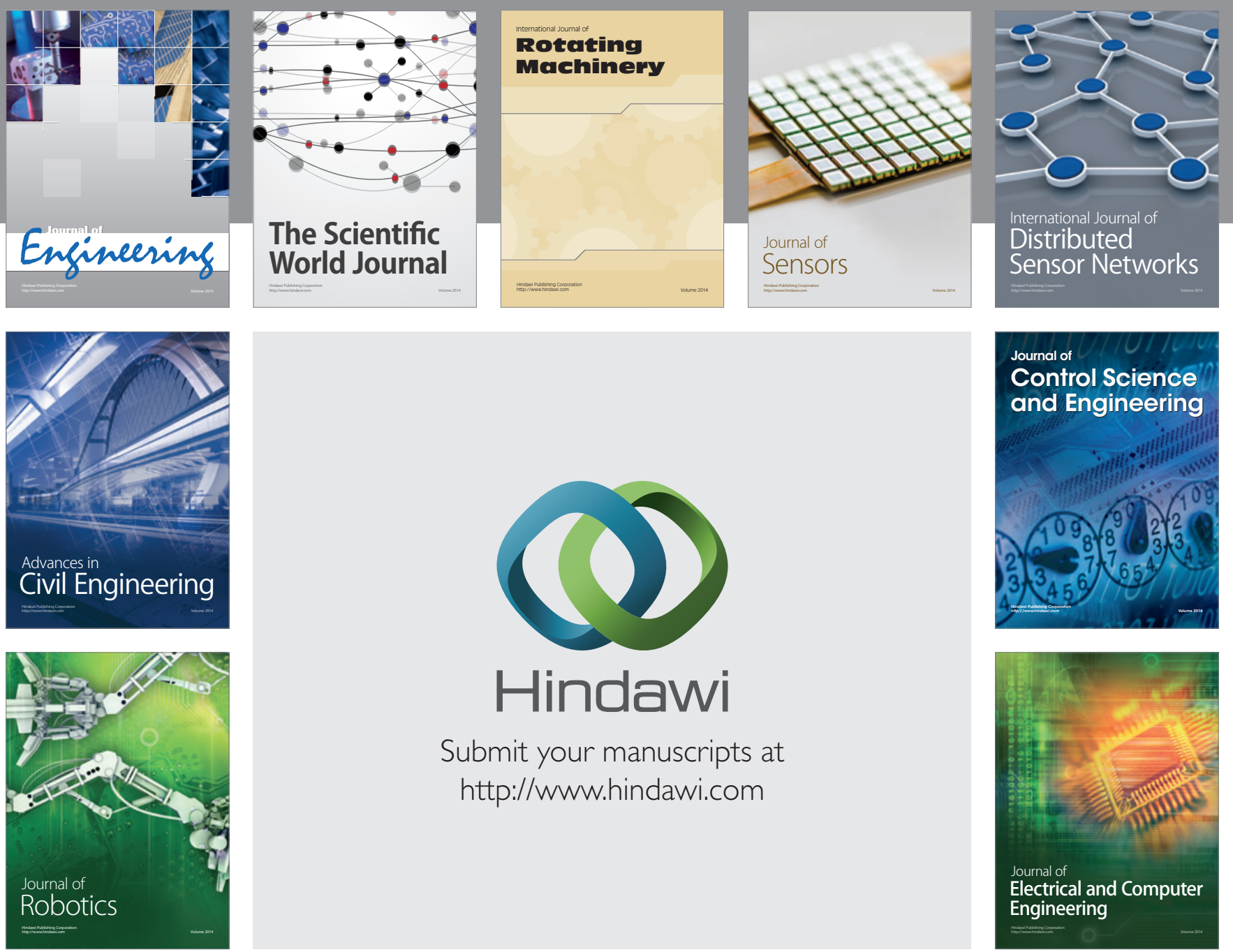

Submit your manuscripts at

http://www.hindawi.com
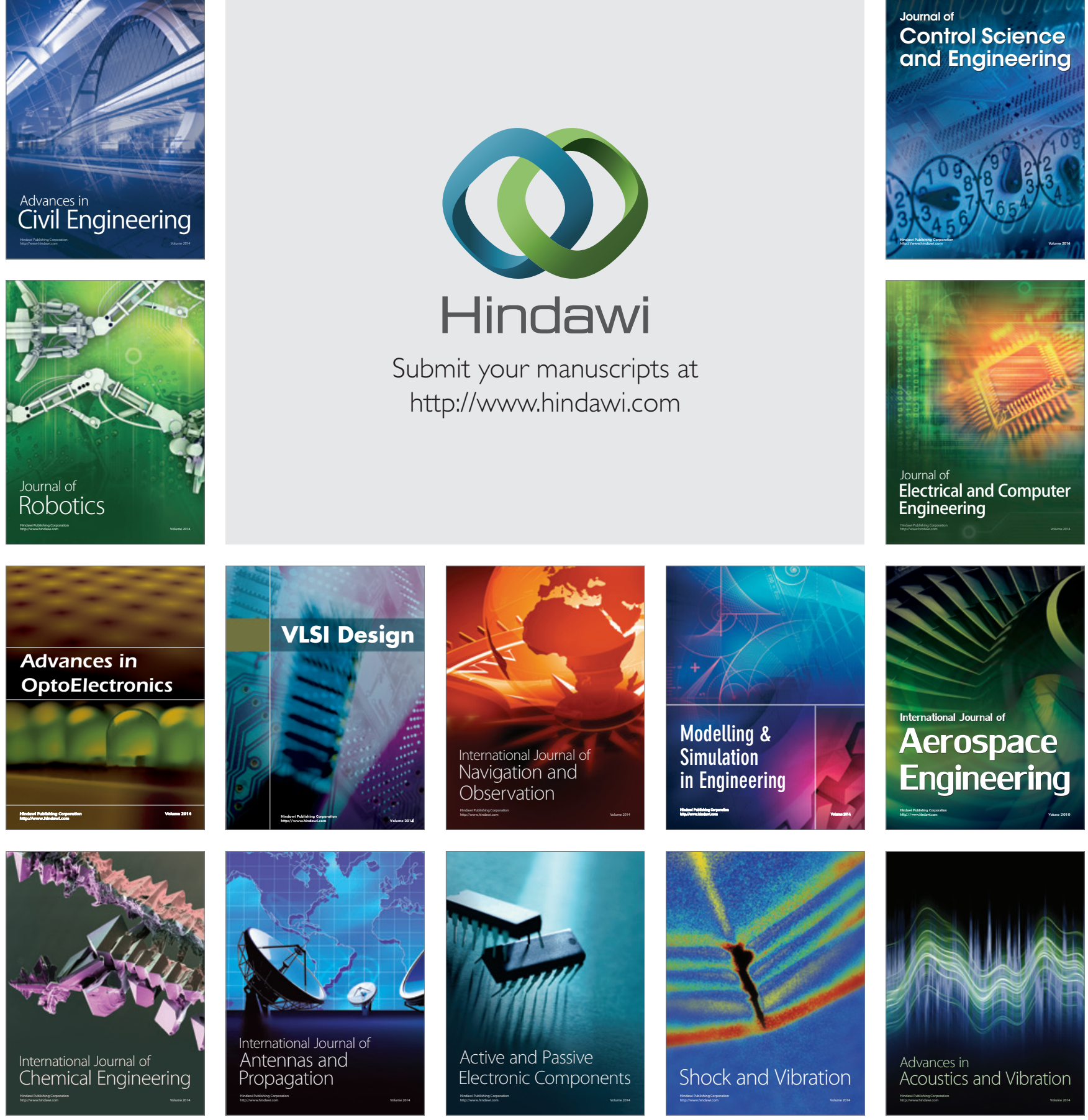